\title{
Hardware design of a submerged buoy system based on electromagnetic inductive coupling
}

\author{
Dalei Song ${ }^{1}$, Shan Gao ${ }^{1}$, Ming Xu ${ }^{2}$, Xiangdong Wang ${ }^{1}$ and Yutao Wang ${ }^{2}$ \\ ${ }^{1}$ Department of Automation, Engineering College, Ocean University of China, Qingdao 266100, China \\ ${ }^{2}$ College of Information Science and Technology, Ocean University of China, Qingdao 266100, China
}

\begin{abstract}
This paper mainly introduces the hardware design of a new type of ocean buoy for multi-scale marine dynamic process. The buoy system can collect a number of real-time marine environment data and then transmit all the data back to the landing site through wireless module. The authors mainly designed the hardware circuit of the buoy system, including data collection system, data communication system, data storage system. Due to the buoy system will complete the marine observation work continuously for at least a month, so we add the low power consumption function which can realize the intermittent work for the data collection system. This paper also introduces the electromagnetic induction coupling technology of underwater sensors, the sea surface communication network technology, etc. The system can also extends to the ecological regional anomaly monitoring and the early warning of disaster weather.
\end{abstract}

\section{Introduction}

As an important support of marine science, marine observation technology is very important to study of marine internal mechanism. In recent years, marine instruments such as ocean turbulence, Argo develops rapidly. Because the marine environment is relatively complex and long-term observation is difficult, some marine observation instruments, such as Argo is chosen the self-contained design [1]. In comparison, the ocean buoy is a very effective method for long-term real-time monitoring of the marine environment. However, most of the observed buoy select one perspective of unilateral physics to observe part of the sea, what's more, most of the message is transmitted based on satellite communication system which has poor real-time performance [2]. This will bring a certain delay for early warning of real-time marine disaster.

This paper introduces a hardware design of a buoy system for multi-scale marine process observation. The buoy system can collect data such as the temperature and velocity from different depth and different sampling point, which uses stable and reliable electromagnetic induction coupling. The communication function of the observation buoy system depends on the point-to-point wireless communication module, and it realizes network construction in the sea using the wireless module in accordance with the self-developed protocol. This paper mainly introduces the hardware design of the buoy system, including real-time data acquisition module for various sensors, multiple storage module, real-time data transmission module etc. Moreover, this paper also puts forwards the low-power mode design of microcontroller, which plays a vital role in the buoy system for long-term submersible deployment.

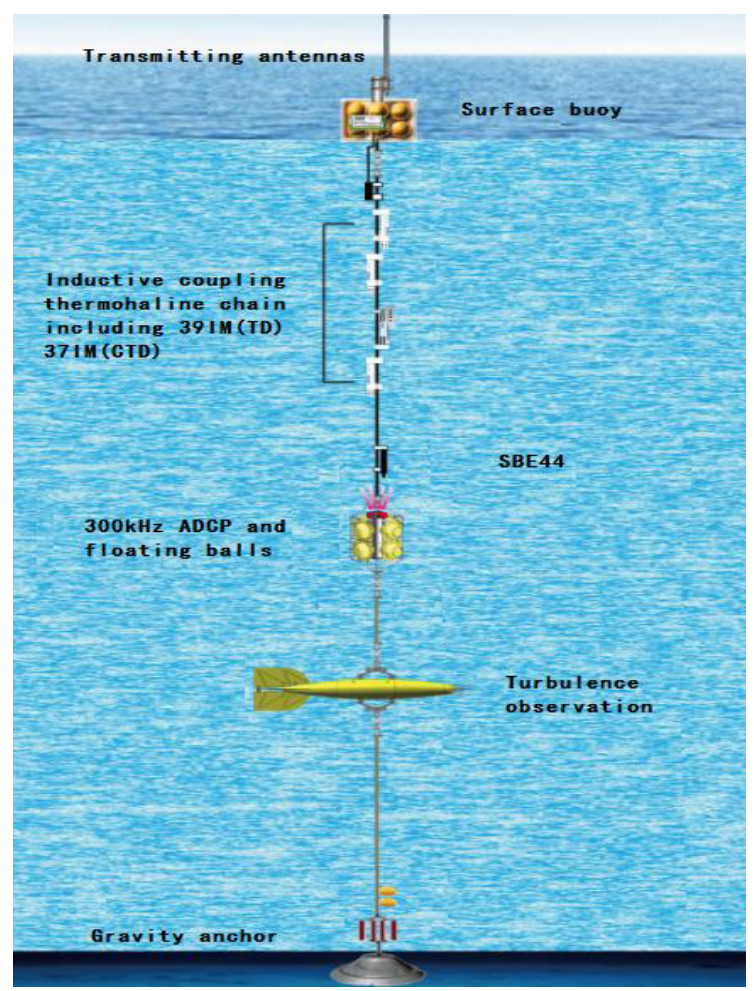

Figure 1. Diagram of the buoy system deployment. 


\section{Design of buoy system}

In order to realize the multi-scale ocean dynamic process observation, the buoy system will carry a variety of sensors, and according to different sampling points, changes in the physical parameters of real-time observations in various regions can be read on shore. The buoy system is mainly composed of the wireless communication device, floating body, a set of Acoustic Doppler Current Profiler, 4 sets of the CTD sensor, 16 sets of the TD sensor, a turbulence instrument, a GPS system and mooring recycling system. Figure 1 shows the deployment of all the sensors of the system.

The buoy system mainly collects real-time TD, CTD, ADCP and GPS data. Among them, TD and CTD sensors all work by electromagnetic induction coupling. Due to the ADCP has no function of inductive coupling, we use SBE44 to achieve transparent transmission. Electromagnetic induction coupling module also includes IMM module, power supply module. In the laboratory test, we use the loop wire to go through IMM coupler. Of course, in order to ensure the reliability of future sea trial, we will use the plastic coated steel pipe instead of loop wire to connect all the sensors. The turbulence observation instrument of the buoy system is designed and tested alone by Ocean University of China [3], which can realize micro-scale marine environment observation. Because of the large amount of marine data, the turbulence observation instrument is self-contained designed and does not participate in the real-time communication. The instrument can simultaneously capture turbulence, three direction acceleration and angular velocity and depth data synchronous sampling. The turbulence observation instrument circuit design is based on ARM7 processor as the core control chip, and the turbulent signal is obtained via a process of charge amplifier, differential, filtering, voltage amplifier and filtering conversion by the shear probe. All the sensors and instruments will be hung in order across the plastic coated steel pipe and then launched in the sea with the mooring recycling system.

The buoy system uses network construction in the sea to communicate with the wireless module on shore. We choose FGR2 wireless communication module to construct network, which mainly contains FGR2 transmission unit, protocol controlled module and antenna module. It depends on its long distance transmission function, strong anti-interference ability and high transmission rate to achieve network construction in the sea. The main principle of the communication method is to use a FGR2 as the master node and the other FGR2 as slave node fixed to each buoy system. The master node will send handshake request packet continuously for 10 times to node network by polling each buoy sequentially. If the slave nodes receive handshake request package, they will respond handshake response package to the master node immediately. As long as the master node received the response more than 5 times, it grants the slave nodes a network access. After adding slave nodes in the network, the master node interact with slave nodes data in accordance with our self-developed network protocol and the specified data format. The slave nodes will send the collected real-time data to the master node, and the master node will parse data at the same time, then the slave node will wait for a next data request from the master node.

\section{Hardware design}

For the hardware design of the buoy system, the most important section consists of two parts. On the one hand, it needs to acquire real-time data of the underwater sensors, including the TD, CTD, ADCP and GPS signals; on the other hand, it needs to transmit collected data to the wireless communication module according to the network construction protocol in accordance with the requirements for a certain period of time. The hardware block diagram of the whole system is shown in figure 2 .

\subsection{Data collection system}

The entire hardware circuit of the data collection control system mainly includes: Stm321151c8t6 microcontroller, MAX3223 serial transceiver module, external flash memory, battery, etc [4]. The control system is mainly composed of MCU Stm321151c8t6 and peripheral circuit, which is the control center of the module. In addition, the system adopts the external flash memory to store collected sensor data. The data acquisition system can exchange data with various sensors through the communication interface.

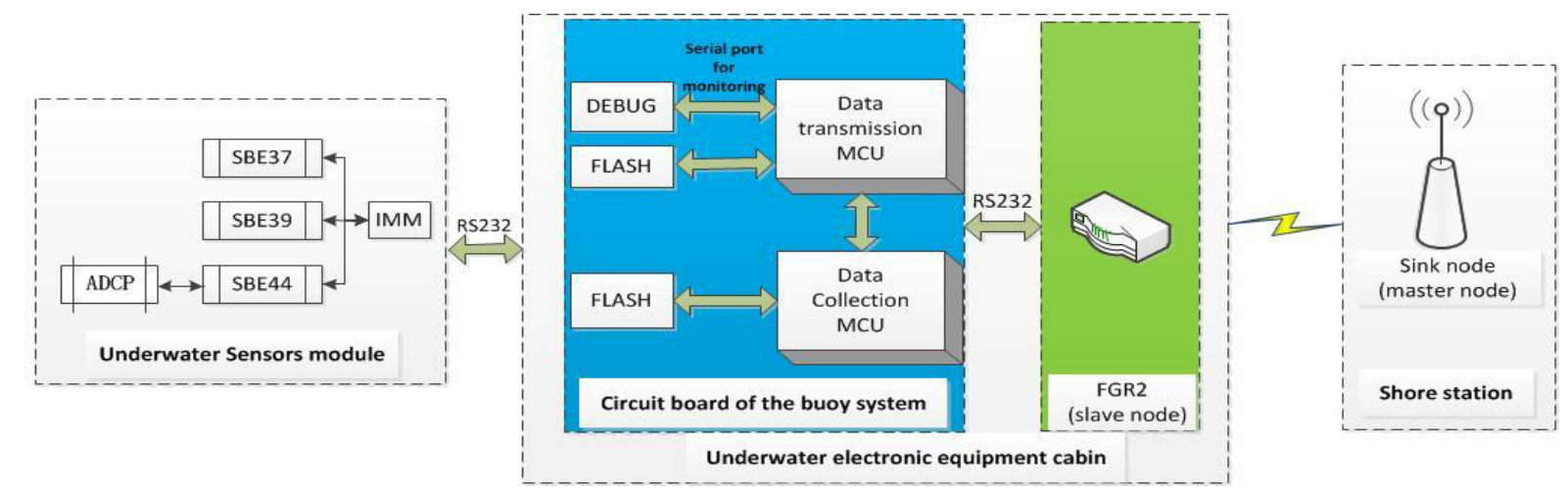

Figure 2. Hardware block diagram of the buoy system. 
The design of data acquisition circuit is completed based on Stm32 series MCU, which model is Stm321151c8t6. In order to satisfy the requirement of the system for long-term submersible deployment, we select the type of microcontroller because it has low power mode. Moreover, the standby current of the microcontroller is only $0.3 \mathrm{uA}$. In addition, the Stm321 series microcontroller is a typical ultra low power and high performance microprocessor which is compatible with $\mathrm{Stm} 32$. It works on $32 \mathrm{MHz}$ and its power supply is $1.65 \mathrm{~V}$ to $3.6 \mathrm{~V}$. It has analog and digital peripherals including watchdog, 12-bit ADC, 12-bit DAC, timer, power supply manager, internal low frequency oscillator, etc. It provides a variety of bus interface, including a USB, three UART, two $16 \mathrm{Mbits} / \mathrm{s}$ SPI, two I2C. And additionally, it has 2 ultra-low-power-comparators and supports serial wire debug, JTAG and trace. It uses the flash ROM technology and has $128 \mathrm{~KB}$ flash ROM, 16KB RAM, up to 83 fast I/Os (73 I/Os $5 \mathrm{~V}$ tolerant), up to $4 \mathrm{~KB}$ of true EEPROM with ECC. It is a system which can work independently.

According to the communication protocol between master node and slave node, the master node request data every 10 minutes and during the 10 minutes, each sensor needs collect data 5 times at each sampling point. All data should be uploaded to the communication module microcontroller after collecting, but before uploading, all data must be written to the external flash memory to guarantee against data loss and compare with the received data from master node conveniently in the future. Due to buoy system needs to work continuously for at least a month, and all data collected by the sensors every 10 minutes is a total of 2190 bytes, according to the results of calculation, we select a 16M flash memory W25Q128. The flash memory supports SPI work mode, it can connect with the microcontroller through interface to realize read and write real-time data.

Electromagnetic induction coupling has the advantages of high reliability and strong stability, therefore, we select the sensors which can work in this way. Among them, TD and CTD can apply to the work mode directly, while the ADCP needs transparent transmission via SBE-44 to use the work mode. When the underwater instruments work, they need insulated wire looped through modem coupling core to connect to the IMM module. The IMM module is a low power microprocessor peripheral that interfaces to inductive mooring by serial RS-232 or RS-485. We select MAX3232 serial transceiver module as the communication mode. We set aside two serial ports when we design the circuit, one is connected with the IMM module and the other is connected with GPS.

In the design of data acquisition circuit, we added the low power consumption mode. According to the demands of the protocol, underwater sensors take two minutes to acquire data for once. Actually, it takes less than two minutes to collect all the data once. So after the acquisition of the data, the data collection system will send commands to make all sensors enter hibernation immediately and the microcontroller enter standby mode at the same time. The control board only needs to provide power for the clock module to reduce the ineffective power dissipation which saves the electricity consumption.

\subsection{Data transmission system}

The hardware circuit of the data transmission control system mainly includes: MAX3232 serial transceiver module, Stm321151c8t6 MCU, external flash memory, voltage conversion module etc. The design of the data transmission control system is mainly to receive data from the data acquisition control system [5]. It also needs to control the slave node when it receives network access request command from the master node. Then the data collected in recent 10 minutes will be uploaded to the master node.

The data transmission module also has a piece of external flash storage which purpose is to store all the data sent to the master node after finishing packing. In this way, the collected data not only can be compared with data which the data collection system gets, also can be compared with received data through the wireless transmission module so as to ensure the accuracy of the data.

The data transmission module is mainly for data exchange with the FGR2 wireless communication module. FGR2 communication module supports RS-232 communication interface, so we set aside a serial port on the circuit board with FGR2. The data transmission control system will send real-time data to FGR2 slave node every 10 minutes via a serial port. If FGR2 master node send data request package to FGR2 slave node, FGR2 slave node will send the last ten minutes data to the FGR2 master node. In addition, we also set aside a serial port for monitoring when we design the circuit board to facilitate the debugging of the system. The serial port can send the data collected, the data transferred and the data stored in two pieces of flash to the computer which realizes the data real-time monitoring and verification. What's more, it also provides great convenience for debugging programs.

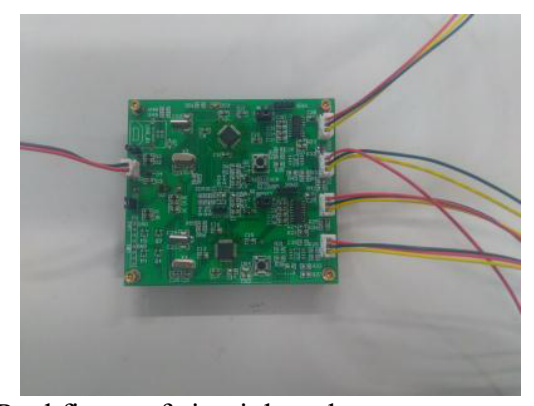

Figure 3. Real figure of circuit board.

Because of limitations of the submergence depth and the limited space of the electronic cabin, the data acquisition circuit and transmission circuit are integrated on a circuit board. Communication between the two pieces of MCU are realized through an IO port. Both IMM module of data gathering and FGR2 module for data transmission adopt RS-232 communication with microcontroller. In order to facilitate the system for long distance transmission in the future, the RS-485 
communication module is also reserved in this dual-core control circuit board. The real figure of the circuit board is shown in figure 3 .

\subsection{Power system}

The hardware circuit of the submersible buoy system needs a total of 4 modules for external power supply. The design voltage of data acquisition and transmission control circuit is $3.6 \mathrm{~V}-6.5 \mathrm{~V}$, the power supply voltage of wireless communication module FGR2 is $12 \mathrm{~V}$, and electromagnetic induction coupling IMM module voltage is between 6 and $30 \mathrm{~V}$, in addition, GPS module needs additional power supply with the voltage of $5 \mathrm{~V}$. Therefore, in order to ensure that multi-scale ocean dynamic process observation system is able to complete at least one month's long-term stability deployment, we choose to add DC-DC converter module and unify with the $12 \mathrm{~V}$ alkaline battery to supply power which reduce the risk on a variety of batteries according to the calculation.

Except the hardware circuit system, the underwater sensors TD, CTD and ADCP all bring supply battery and can work continuously more than 6 months. The turbulence instrument also comes with batteries without the need for additional battery power supply.

\section{Laboratory tests}

The function of data acquisition and communication control circuit board was tested in the laboratory. The data collection module successfully collected data of 16 TD sensors, 4 CTD sensors, 1 ADCP sensor and GPS, and the data collected was stored into the flash while also uploaded to the data communication module at the same time. Moreover, the collected data from each sensor can also be monitored by the serial port in real time, the transfer rate of the serial port is $115200 \mathrm{bps}$.

At the same time, we tested the communication effect between the data communication module and FGR2 module, the network construction effect of FGR2 master node and slave nodes in the laboratory. At the beginning of the experiments we found that the network access effect for the slave node is not very good, what's more, our slave node was often kicked off by the master node because of the slow data transmission. After updating the firmware and modification, it can enter the network smoothly. We also test the packet transmission peak rate, channel rate and so on to prove its stability and the communication effect of the FGR2 module with antenna and without antenna in respectively to simulate poor sea conditions.

The test scene photo was shown in figure 4 . In order to facilitate the test of the whole system, we put the data collection and communication control circuit board, FGR2 communication module and GPS module in a test box and unified with a $12 \mathrm{~V}$ lithium battery to supply power. In laboratory tests, we took a $5 \mathrm{~m}$ long wire instead of the plastic coated steel pipe to complete induction coupling test. Although the data collection and communication control circuit board is unstable at first, some problems may occur like system crashed. We redesigned several versions and the last version has ran for a month stability after improvement. Acquisition data of temperature by 16 TD sensors in ten minutes was shown in figure 5, there was no electrical conductivity and flow velocity data due to the test was operated in the lab.

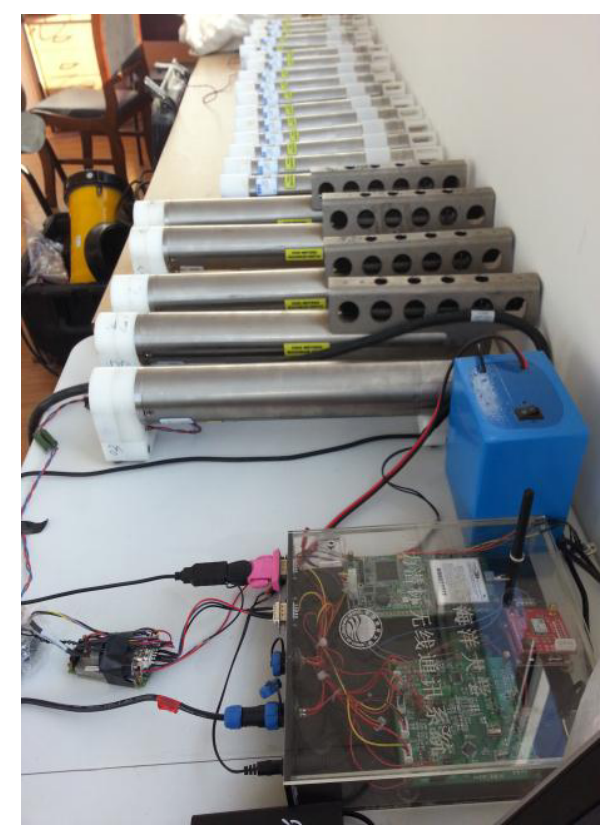

Figure 4. Test scene of system combined adjusting.

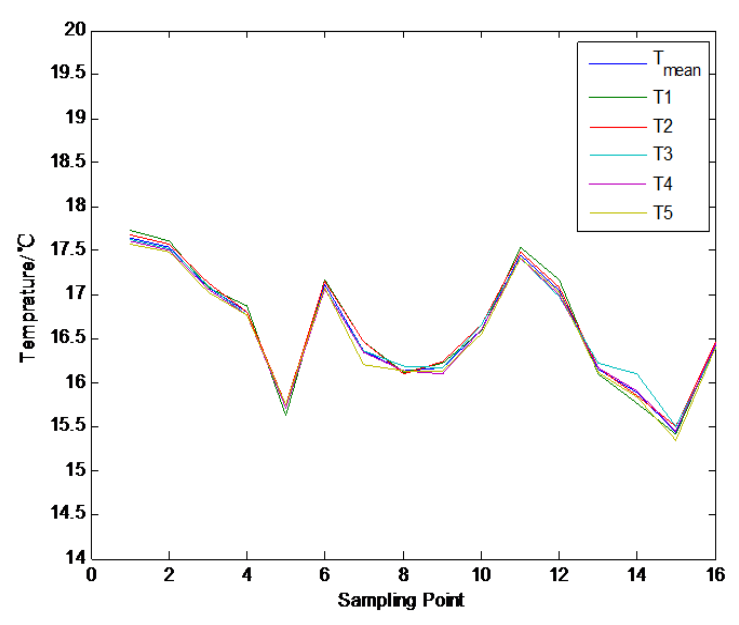

Figure 5. Lab temperature collected by TD sensors.

\section{Conclusion and future work}

This article aims to introduce the hardware design of a buoy system for marine dynamic process observation and the system of the control circuit board has worked accumulated for a month through the test which verified the stability of the hardware system and achieved the design function requirement, it also provides for the buoy system strong support which can maintain a long time deployment in the future. All of the sensors data we collected has carried on the comparison with the real environment in the month, we found that the data are 
normal and discrete degree is small. The stored data in the circuit board has been compared with the data received in the landing site to ensure the consistency of the data. And the hardware system is not only used in this buoy system, it also can be applied to other similar underwater sensor data acquisition system after further development, including long distance communication mode like satellite communication.

Now we are testing the software part of the buoy system. Especially, we test it to communicate with the landing site together with other buoy system and upload real-time collected data to several servers to parse the data. In addition to complete the function indexes, we will test communication status in bad communication condition to simulate poor sea condition as far as possible in order to ensure that the sea trials would be able to complete in the future.

\section{References}

1. Liu, Chun-hu, and B. Fu. Development of a Compact Marine Environmental Monitoring Module Based on
SMT32[C]. Engineering and Technology (S-CET), 2012 Spring Congress on IEEE, 2012:1-4.

2. Zhang H, Liu J, Yu H. Design of New Marine Environmental Data Acquisition and Transmission System $[\mathrm{C}] / / \quad$ Applied Informatics and Communication - International Conference, Icaic 2011, Xi'an, China, August 20-21, 2011, Proceedings. 2011:422-429.

3. Song Da-lei, Sun Jing-jing, Xue Bing, et al. Mooring system of ocean turbulence observation based on submerged buoy $[\mathrm{J}]$. China Ocean Engineering, vol.27, pp 369-378,2013.

4. Liu S H, Gong D J, Yong-Ping X U, et al. The control circuit of the marine environment monitoring system based on microcontroller[J]. Marine Sciences, 2009.

5. Zhang Y, Cai W. The Marine Environment Monitoring Sensor Network Research Based on the Coupling Communication Chain[C]// International Conference on Control Engineering and Communication Technology. 2012:358-361. 